\title{
Research on Protein Level in Medical Latex Glove Images using Color Kernel Regression Method
}

\author{
CK Toa, KS Sim, JLTan
}

\begin{abstract}
In the healthcare environment, medical latex gloves are a necessary medical item for healthcare workers as it offers excellent hand barrier protection against dangerous microorganism. However, if the healthcare workers repeated exposure to the latex gloves which contain high protein level, it will increase the possibility of the workers to have a risk for latex allergy. Thus, the objective of this project is to develop a color kernel regression (CKR) method for estimating protein level through the analyses of color difference in glove images. Initially, the gloves will go through an uncomplicated chemical test for protein detection. A blue color will appear on the surface of a glove sample that contains protein. After that, the chemical binded sample will be digitally converted into a sample image using the flatbed scanner. The image will then undergo image processing to improve its quality and to calculate the color difference values of the sample. Those calculated values with the pre-defined protein levels will be used to plot a standard graph. A high coefficient of determination with $R 2>98 \%$ has been obtained from the experimental graph. This indicates that the proposed CKR method contributes significantly toward the estimation of protein level.
\end{abstract}

Index Terms - Color difference, Color kernel regression, Latex allergy, Medical latex gloves, Protein level

\section{INTRODUCTION}

Medical latex gloves are made of Natural Rubber Latex (NRL) which offers few attractive properties such as tear resistance, barrier protection, and elasticity quality [1]. Those gloves have been used widely in the healthcare sector as they can provide protection to the user against a wide range of bacteria [2]. The only problem encounter with the latex glove is the protein that remains on the glove. A high level of protein will increase the possibility of the user to have a risk for latex allergy [3], [4]. In order to estimate the protein level of the glove, several conventional methods such as Lowry method and Bradford protein assay have been developed. Those methods determine protein of the glove through a chemical test to observe the color change in the solutions [5], [6]. Those solutions will then be analyzed using the spectrometer to estimate the protein level. Basically, those conventional methods were more into the chemical analysis as it involved with numerous chemical reagent and expensive equipment. As a result, those

Revised Manuscript Received on August 14, 2019.

CK Toa, Faculty of Engineering and Technology, Multimedia University, Jalan Ayer Keroh Lama, 75450 Bukit Beruang, Melaka, Malaysia. (E-mail: toacheankhim@yahoo.com)

KS Sim, Faculty of Engineering and Technology, Multimedia University, Jalan Ayer Keroh Lama, 75450 Bukit Beruang, Melaka, Malaysia. (E-mail: kssim@mmu.edu.my)

JLTan, Faculty of Engineering and Technology, Multimedia University, Jalan Ayer Keroh Lama, 75450 Bukit Beruang, Melaka, Malaysia. (E-mail: tanjinlong95@gmail.com) methods were complicated in the chemical test, time consumption and costly.

The objective herein is to propose a new method with comparative advantage to the conventional methods by focusing on digitizing the protein estimation process in order to reduce involvement in chemical analysis. In this paper, a computerized method named color kernel regression (CKR) has been proposed. This method consists of two processes which are a chemical test and digital color image processing. The first process detects the protein inside the glove sample through an uncomplicated chemical test, while the second process converts the glove sample into a digital color image to perform image processing, calculate the color difference value and plot the standard curve.

This paper is arranged as follows, protein estimation methods are discussed in section II. The results and discussion of the image processing, color difference value, and analysis of the graph will be elaborated in section III. The summary of the proposed method will be in the conclusion section.

\section{PROTEIN ESTIMATION METHODS}

\section{A. Bradford microplate protein assay method}

The Bradford assay [7], [8] can be used to quantify the protein of the latex glove. The basic mechanism of the assay is the binding of the Bradford reagent at acidic $\mathrm{pH}$ to an amino acid found in protein [9]. If the solution contains protein, the color of the solution will change from brown to blue. The blue color solution will be measured by the spectrometer to calculate absorbance value. The process of a chemical test for Bradford microplate protein assay is shown in Fig.1. 


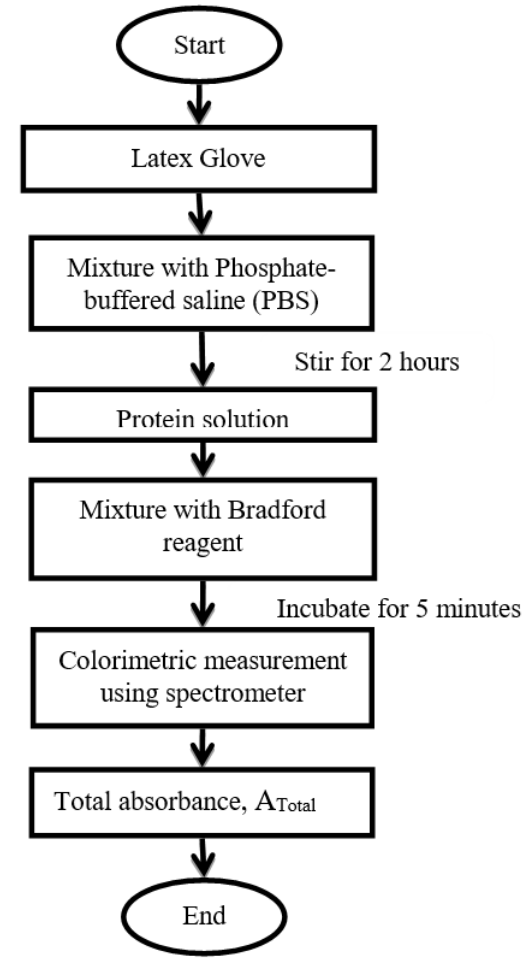

Fig.1: Process of Bradford microplate protein assay

After going through the chemical process [10], the absorbance data will be plotted in the standard curve as shown in Fig. 2 to estimate the protein level.

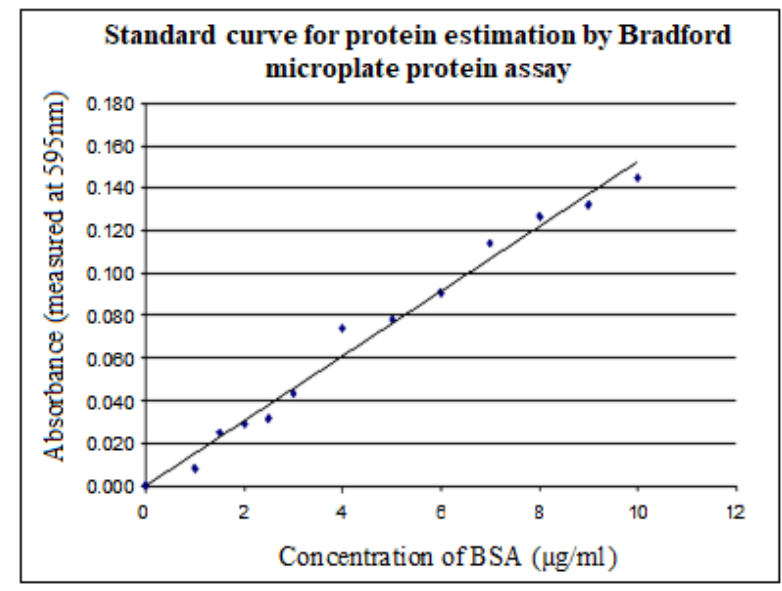

Fig.2: Standard curve of Bradford assay

Based on the process of the chemical test, it is tedious since the extraction of the protein solution from the glove was time-consuming. Furthermore, the device such as spectrometer that used to perform the colorimetric measurement is costly and unaffordable to purchase by the students for academic purpose.

\section{B. Proposed method}

The Color Kernel Regression (CKR) method was proposed to estimate the protein level of the latex glove. The CKR method focuses on digitizing the protein estimation process with comparative advantage to the Bradford protein assay method. Fig.3 shows the flow chart of the CKR method.

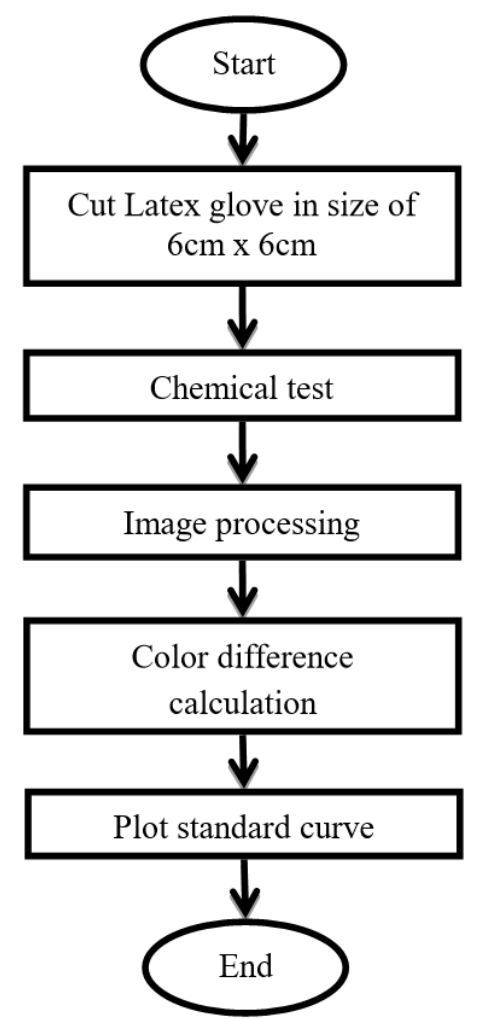

Fig.3: Flow chart of the Color Kernel Regression (CKR) method

\section{Chemical test}

The procedure of the chemical test is shown in Fig.4. Firstly, the glove sample that cut into a size of $6 \mathrm{~cm}$ by $6 \mathrm{~cm}$ will immerse into the Bradford assay [11], [12] for 15 minutes. If the glove sample contains protein, the Bradford assay will bind with it, converting the white color of the sample into a blue color. After that, the chemical binded sample will be risen using distilled water to remove excess chemical reagent.

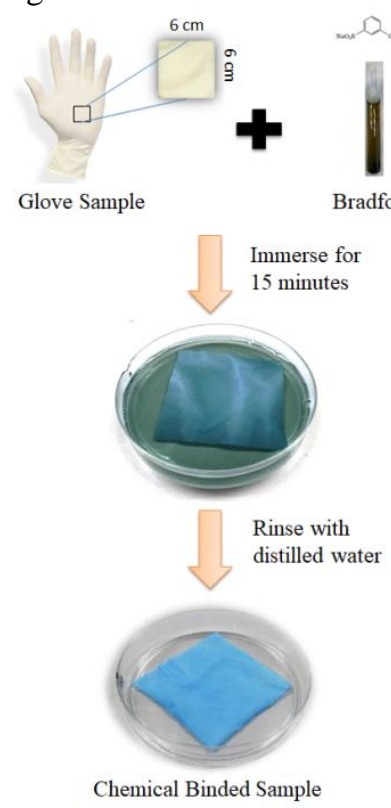

Fig.4: Process of the chemical test 


\section{Image processing}

After the chemical test, the chemical binded sample will be scanned using a flatbed scanner to convert the sample into a digital color image as shown in Fig.5.

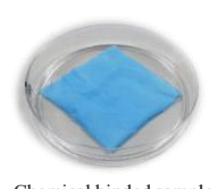

Chemical binded sample

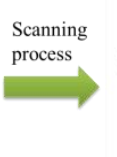

Fig.5: Process of image acquisition
Later, the chemical binded sample image will go through image processing to filter out the crease on the glove surface. The appearance of the crease will negatively affect the calculation of the color difference in the sample image. Thus, a new technique named Adaptive Thresholding Mean Filtering (ATMF) has been proposed to filter out the crease on the sample surface as shown in Fig.6. The following algorithm shows the calculation of the ATMF technique.

Algorithm 1: Adaptive Thresholding Mean Filtering (ATMF)

1: $\quad$ Separate the sample image, $\mathrm{I}_{\mathrm{S}}(\mathrm{x}, \mathrm{y})$ into the red, green, and blue color channel. Convert those channels into a histogram respectively.

2: $\quad$ Find the starting threshold, $\mathrm{T}_{\mathrm{S}}$ by finding the mean of the histogram.

3: $\quad$ Using the $\mathrm{T}_{\mathrm{S}}$ as the reference point, separate the histogram into two groups: $\mathrm{G}_{1}$ and $\mathrm{G}_{2}$. Calculate the mean values, $\mathbf{M}_{1}$ (object) and $\mathbf{M}_{2}$ (background) of the groups, $\mathrm{G}_{1}$ and $\mathrm{G}_{2}$ by using (1) and (2).

$$
\begin{gathered}
M_{1}=\frac{\sum_{(i, j)} G_{1}(i, j)}{\text { Total object pixels }} \\
M_{2}=\frac{\sum_{(i, j)} G_{2}(i, j)}{\text { Total background pixels }}
\end{gathered}
$$

4: Calculate the average value of $\mathrm{M}_{1}$ and $\mathrm{M}_{2}$ to find the new threshold, $\mathrm{T}_{\text {New }}$ as shown in (3).

$$
T_{N e w}=\frac{1}{2}\left(M_{1}+M_{2}\right)
$$

5: $\quad$ If $\mathrm{T}_{\mathrm{New}} \neq \mathrm{T}_{\mathrm{S}}$, return to step 3 and the new value of $\mathrm{T}_{\mathrm{S}}$ $=\mathrm{T}_{\mathrm{New}}$, otherwise proceed next step.

6: $\quad$ Replace each pixel in the image with white pixel if $\mathrm{I}_{\mathrm{S}}(\mathrm{x}, \mathrm{y})<\mathrm{T}_{\mathrm{New}}$ and black pixel if $\mathrm{I}_{\mathrm{S}}(\mathrm{x}, \mathrm{y}) \geq \mathrm{T}_{\text {New }}$. The white pixel represents the creased region, $\mathrm{I}_{\text {creased }}(\mathrm{x}, \mathrm{y})$, while the black pixel represents the background region, $\mathrm{I}_{\text {background }}(\mathrm{x}, \mathrm{y})$.

7: Calculate the mean value of background sample, $\left.\mathrm{I}_{\text {background }}^{*} \mathrm{x}, \mathrm{y}\right)$ by using (4).

$$
I_{\text {background }}^{*}(x, y)=\frac{\sum_{(x, y)} I_{\text {background }}(x, y)}{\text { Total black pixels }}
$$

8: $\quad$ Substitute $\mathrm{I}_{\text {creased }}(\mathrm{x}, \mathrm{y})$ to the value of $\mathrm{I}_{\text {background }}(\mathrm{x}, \mathrm{y})$ for filtering out the crease region.

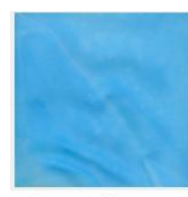

Input Image
RGB Histogram

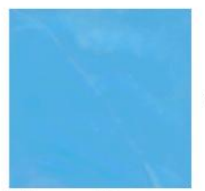

Filtered Image

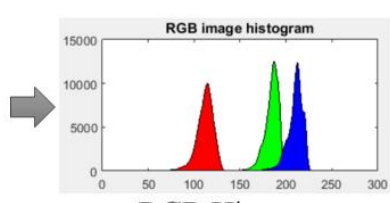

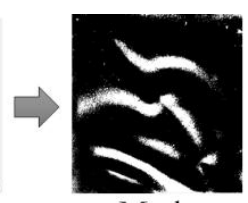

Mask

Adaptive

Thresholding

Mean Filtering

(ATMF)

Filtering process
Fig.6: Process of crease filtering on the sample using the ATMF technique

\section{Color Difference Calculation}

After obtained filtered image, the next step is to calculate the color difference delta $\mathrm{E}, \Delta \mathrm{E}$ value between the raw image (Before chemical test) and chemical binded image (After chemical test). Fig.7 shows flow chart of the color difference calculation.

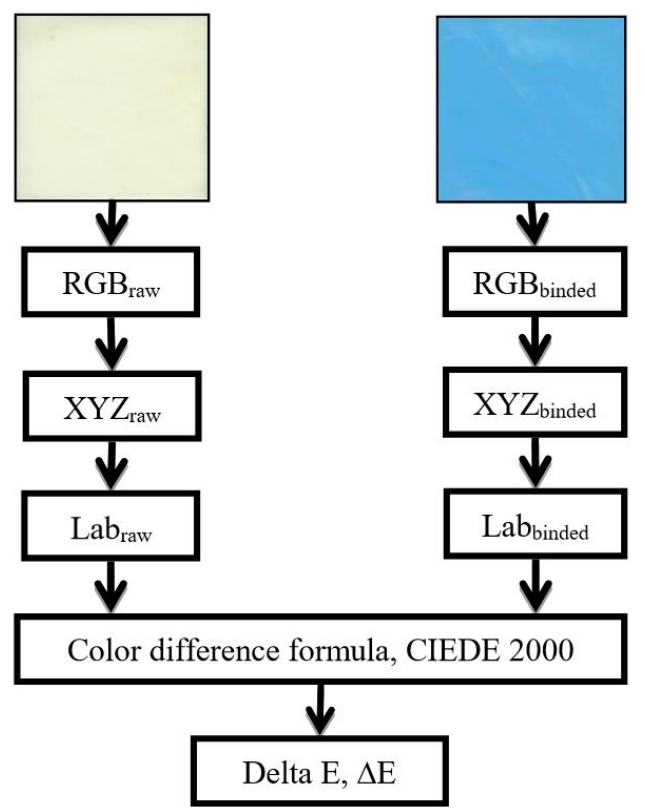

Fig.7: Flow chart of the color difference calculation

Based on Fig.7, the RGB value of the raw image and the chemical binded image will convert into XYZ tristimulus value [13] by using (7).

$$
\begin{gathered}
D \in\{R, G, B\} \\
D^{\prime}= \begin{cases}\frac{D}{12.92} & \text { for } D \leq 0.04045 \\
\left(\frac{(D+0.055)}{1.055}\right)^{2.4} & \text { for } D>0.04045\end{cases} \\
{\left[\begin{array}{l}
X \\
Y \\
Z
\end{array}\right]=\left[\begin{array}{ccc}
0.41245564 & 0.3575761 & 0.1804375 \\
0.212672 & 0.7151522 & 0.0721750 \\
0.01933399 & 0.1191920 & 0.9503041
\end{array}\right]\left[\begin{array}{l}
R^{\prime} \\
G^{\prime} \\
B^{\prime}
\end{array}\right]}
\end{gathered}
$$


where D consists of RGB color value and $D$ is the linearization of $\mathrm{D}$. Later, the XYZ value will be converted into International Commission on Illumination (CIE) Lab [14] by using (8), (9), and (10).

$$
\begin{gathered}
L^{*}=\left\{\begin{array}{rr}
116\left(7.787 \frac{Y}{N_{Y}}+0.138\right)+16 & \text { for } \frac{Y}{N_{Y}} \leq 0.008856 \\
116 \sqrt[3]{\frac{Y}{N_{Y}}-16} & \text { for } \frac{Y}{N_{Y}}>0.008856
\end{array}\right. \\
a^{*}=\left\{\begin{array}{r}
500\left[\left(7.787 \frac{X}{N_{X}}+0.138\right)-\left(7.787 \frac{Y}{N_{Y}}+0.138\right)\right] \text { for } \frac{X}{N_{X}} \leq 0.008856 \\
500\left[\sqrt{\frac{X}{N_{X}}}-\sqrt[3]{\left.\frac{Y}{N_{Y}}\right] \text { for } \frac{X}{N_{X}}>0.008856}\right.
\end{array}\right. \\
b^{*}=\left\{\begin{array}{r}
200\left[\left(7.787 \frac{Y}{N_{Y}}+0.138\right)-\left(7.787 \frac{Z}{N_{Z}}+0.138\right)\right] \text { for } \frac{Z}{N_{Z}} \leq 0.008856 \\
200\left[\frac{Y}{N_{Y}}-\sqrt[3]{\left.\frac{Z}{N_{Z}}\right]} \text { for } \frac{Z}{N_{z}}>0.008856\right.
\end{array}\right.
\end{gathered}
$$

where $\mathrm{N}_{\mathrm{X}}, \mathrm{N}_{\mathrm{Y}}$, and $\mathrm{N}_{\mathrm{Z}}$ are the normalized $\mathrm{XYZ}$ value. After that, the $L^{*}, a^{*}$, and $b^{*}$ value will substitute into the formulation of CIEDE2000 [15], [16] to calculate the delta $\mathrm{E}$ as shown in (14).

$$
\begin{gathered}
\Delta L^{*}=L_{\text {binded }}^{*}-L_{\text {raw }}^{*} \\
\Delta C^{*}=\left(a_{\text {binded }}^{* 2}+b_{\text {binded }}^{* 2}\right)^{0.5}-\left(a_{\text {raw }}^{* 2}+b_{\text {raw }}^{* 2}\right)^{0.5} \\
\Delta h^{*}=2 \sqrt{\left(a_{\text {raw }}^{* 2}+b_{\text {raw }}^{* 2}\right)^{0.5} \cdot\left(a_{\text {binded }}^{* 2}\right)^{0.5}} \sin \left(\frac { 1 } { 2 } \left(\tan ^{-1}\left(\frac{b_{\text {binded }}^{*}}{a_{\text {binded }}^{*}}\right)\right.\right. \\
\left.\left.-\tan ^{-1}\left(\frac{b_{\text {raw }}^{*}}{a_{\text {raw }}^{*}}\right)\right)\right) \\
\Delta E=\sqrt{\left(\frac{\Delta L^{*}}{S_{L}}\right)^{2}+\left(\frac{\Delta C^{*}}{S_{C}}\right)^{2}+\left(\frac{\Delta h^{*}}{S_{H}}\right)^{2}+R\left(\frac{\Delta C^{*}}{S_{C}}\right)\left(\frac{\Delta h^{*}}{S_{H}}\right)}
\end{gathered}
$$

$\Delta \mathrm{L}^{*}, \Delta \mathrm{C}^{*}, \Delta \mathrm{h}^{*}$ are lightness, chroma, and hue difference value, $S_{L}, S_{C}, S_{H}$ are the weighting functions and $R$ is a rotational function.

\section{RESULT AND DISCUSSION}

In this experiment, over a hundred glove samples have been tested for protein estimation. Those samples with crease surface will go through Adaptive Thresholding Mean Filtering (ATMF) technique for filtering process and the result is shown in Table I.

Table I: Image filtering using (ATMF) technique

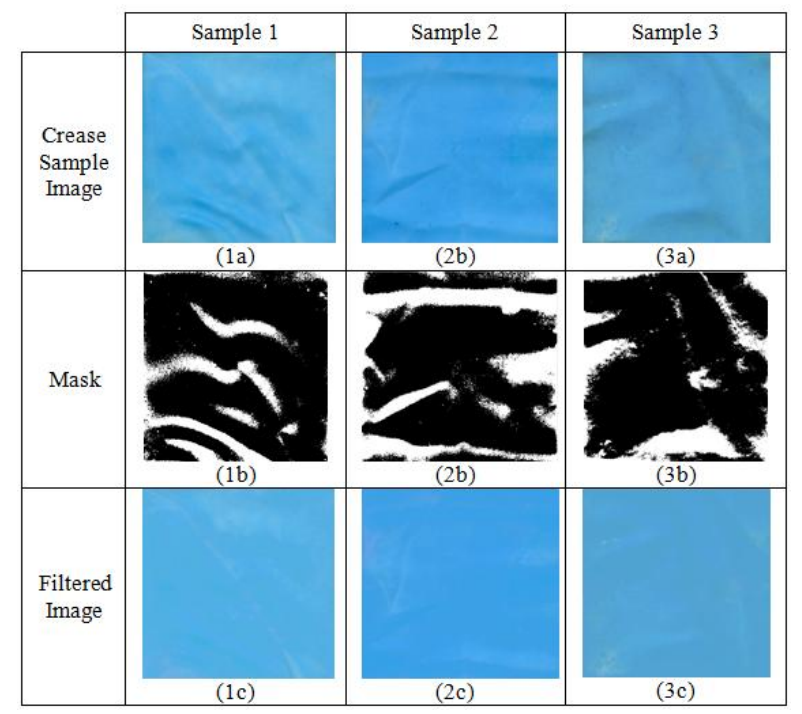

Based on the images in Table I, it shows that there have no creases remain in the filtered image. This is proven that the ATMF technique is performing well on filtering out the crease in the sample image. Moreover, to verify that the ATMF technique able to restore important information from crease image, we will calculate the absolute error of color difference for Crease-Free image $\left(\Delta \mathrm{E}_{\text {Crease-Free }}\right)$, Crease image $\left(\Delta \mathrm{E}_{\text {Crease }}\right)$, and Filtered image $\left(\Delta \mathrm{E}_{\text {Filtered }}\right)$ using (15) and (16).

$$
\begin{aligned}
& \text { Absolute Error } \text { crease }=\Delta E_{\text {Crease-Free }}-\Delta E_{\text {Crease }} \\
& \text { Absolute Error Filtered }=\Delta E_{\text {Crease-Free }}-\Delta E_{\text {Filtered }}
\end{aligned}
$$

A bar chart has been plotted in Fig. 8 to show the absolute error of color difference for the crease image and filtered image.

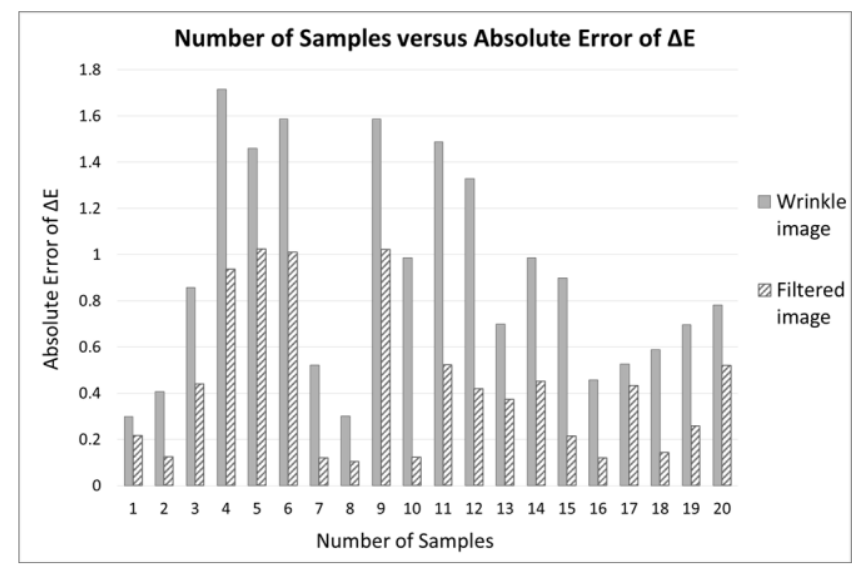

Fig.8: Absolute error for crease image and filtered image

Based on Fig.8, it seems that the absolute error value for the filtered image was smaller than the crease image. Since the absolute error value is smaller, it shows that the $\Delta \mathrm{E}_{\text {Filtered }}$ of the filtered image is approximate to the $\Delta \mathrm{E}_{\text {Crease-Free }}$ of the crease-free image. This indicates that the ATMF technique is not only able to filter out the crease surface but is also capable to restore the important information from crease image. After the filtering process, the color of the filtered sample image will be analyzed to determine the protein level. Based on the analysis result, those samples will be categorized into low level, medium level, and high level of protein as shown in Table II. 
Table II: Three difference protein levels of the sample image

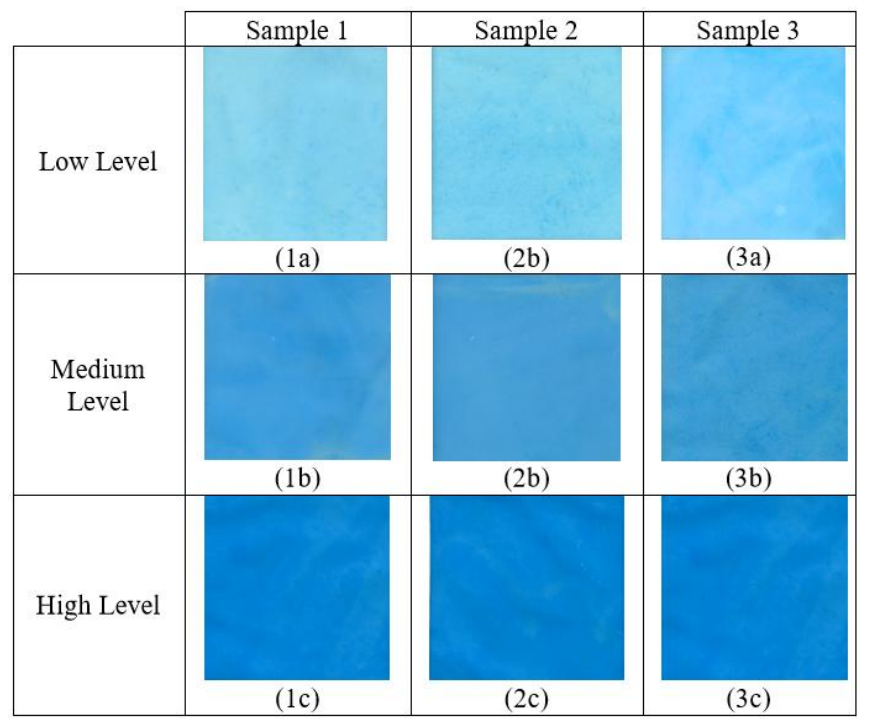

Based on Table II, it can clearly see that different color saturation of the sample image resulting in a different level of protein. For low protein level, the images appear to be light blue color, while high protein level resulting in dark blue color. To verify the proposed protein estimation process, the $\Delta \mathrm{E}$ values, and the pre-defined protein concentration values of those sample images will be plotted into a graph as shown in Fig.9. The pre-defined protein concentration data were obtained from Malaysia Rubber Board (MRB).

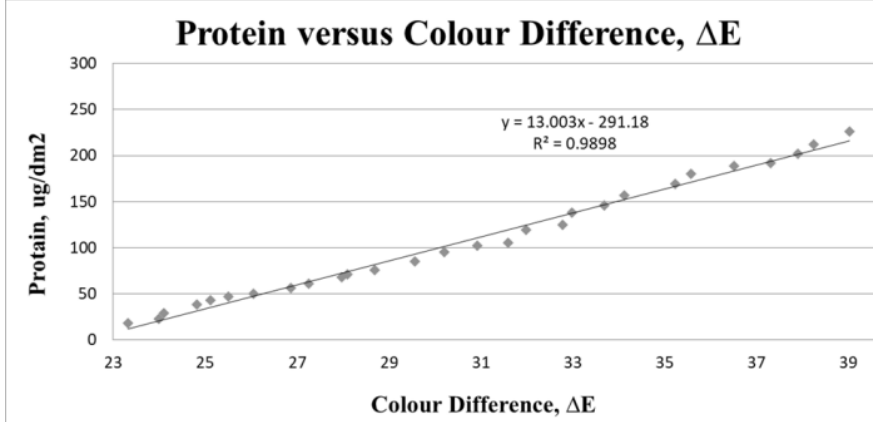

Fig.9: Standard curve for protein concentration and color difference delta $\mathbf{E}$

Based on the graph in Fig.9, it shows that when the $\Delta \mathrm{E}$ value increases, the protein value also increases. This indicates that the protein level can be identified based on the color difference value. By using this graph, we can estimate the protein of unknown sample through the calculation of its color difference $\Delta \mathrm{E}$ value. Besides, with $\mathrm{R}$-squared $\left(\mathrm{R}^{2}\right)$ more than $98 \%$, we can verify that our proposed method has high accuracy on estimating the protein of the glove sample.

\section{CONCLUSION}

Compare to the conventional methods, our proposed Color Kernel Regression (CKR) method able to provide a simple and efficient protein estimation analysis on the latex glove. For the image filtering, the Adaptive Thresholding Mean Filtering (ATMF) technique not only able to filter out the crease on the sample surface but also can restore important information from the crease image. Moreover, the experimented result shows that the standard curve able to perform well on estimating the protein level of an unknown sample with the accuracy of more than $98 \%$. In conclusion, the (CKR) method has a great capability in estimating the protein level based on the color difference of the glove sample.

\section{REFERENCES}

1. E. Yip and P. Cacioli, The manufacture of gloves from natural rubber latex, vol. 110. 2002.

2. M. Mansouri, M. Tidley, K. A. Sanati, and C. Roberts, "Comparison of blood transmission through latex and nitrile glove materials," no. January, pp. 205-210, 2010

3. P. M Ranta and D. Ownby, A Review of Natural-Rubber Latex Allergy in Health Care Workers, vol. 38. 2004.

4. A. Oomman and S. Oomman, Latex glove allergy: The story behind the "invention" of the surgical glove and the emergence of latex allergy, vol. 3. 2013.

5. M. Taghizadeh-Behbahani, B. Hemmateenejad, and $\mathbf{M}$. Shamsipur, "Colorimetric determination of acidity constant using a paper-based microfluidic analytical device," Chem. Pap., vol. 72, no. 5, pp. 1239-1247, 2018.

6. T. Yagami, M. Sato, and A. Nakamura, Colorimetric determination of the total protein eluted from latex gloves. 1993.

7. R. V. Nouroozi, M. V. Nouroozi, and M. Ahmadizadeh, "Determination of Protein Concentration Using Bradford Microplate Protein Quantification Assay," Int. Electron. J. Med., vol. 4, no. 1, pp. 11-17, 2015.

8. J. D. Easterbrook, T. Shields, S. L. Klein, and G. E. Glass, "Smartphone for Point-of-Care Quantification of Protein by Bradford Assay Camila," J. Braz. Chem. Soc, vol. 22, no. 12, pp. 2396-2402, 2011.

9. A. A. Yanos, M. N. Bautista, M. R. N. Angelia, and E. J. Rosario, "Digital Photometric Determination of Protein Using Biuret, Bradford and Bicinchoninic Acid Reagents," vol. 6, no. 2, pp. 168-175, 2013.

10. A. Kumar and S. Koirala, Protein Quantification by Kjeldahl And Bradford Assay Methods. 2018.

11. N. Kruger, "The Bradford Method For Protein Quantitation," in The Protein Protocols Handbook, 2002, pp. 15-21.

12. H. Y. Ting, K. P. Yong, K. L. Mok, and K. S. Sim, "Downhill Search-Based Nrl Glove Protein Estimation," Rubber Chem. Technol., vol. 86, no. 4, pp. 653-663, 2013.

13. S. Bianco, F. Gasparini, A. Russo, and R. Schettini, A New Method for RGB to XYZ Transformation Based on Pattern Search Optimization, vol. 53. 2007.

14. K. León, D. Mery, F. Pedreschi, and J. León, "Color measurement in $\mathrm{L}^{*} \mathrm{a} * \mathrm{~b} *$ units from RGB digital images," Food Res. Int., vol. 39, no. 10, pp. 1084-1091, 2006.

15. Y. Yang, J. Ming, and N. Yu, "Color image quality assessment based on CIEDE2000," Adv. Multimed., vol. 2012, 2012.

16. M. E. Sayed, F. Sammani, and M. A. M. Albashier, "An accurate method to calculate the color difference in a single image," in 2017 International Conference on Robotics, Automation and Sciences (ICORAS), 2017, pp. 1-3. 